\title{
El análisis del discurso como técnica de investigación cualitativa y cuantitativa en las ciencias sociales
}

\author{
DisCOURSE ANALYSIS AS A QUALITATIVE AND QUANTITATIVE TECHNIQUE IN THE SOCIAL SCIENCES
}

Dr. Sebastián Sayago (sebaeldediadema@gmail.com) Escuela de Periodismo, Pontificia Universidad Católica de Valparaíso (Valparaíso, Chile)

\begin{abstract}
This article proposes that Discourse Analysis (DA) be methodologically characterized as an analytical technique for the social sciences. To do this, it must first be situated in relation to two other methodological tools used for the study of discourse: hermeneutics and Content Analysis. Subsequently, the article will define DA, focusing on one aspect in particular: its compatibility with both qualitative and quantitative research strategies. It will then examine the usefulness of this technique in the process of data construction and finally propose an assessment of this issue.
\end{abstract}

Keywords: discourse analysis, methodology, qualitative research, quantitative research

\begin{abstract}
Resumen
En este artículo nos proponemos caracterizar metodológicamente al Análisis del Discurso (AD) en tanto técnica de análisis de las ciencias sociales. Para ello, primero, lo ubicaremos en relación con otras dos herramientas metodológicas utilizadas para estudiar el discurso: la hermenéutica y el análisis de contenido. Posteriormente, haremos una caracterización del $A D$, centrándonos en un aspecto en particular: su compatibilidad tanto con estrategias cualitativas como con estrategias cuantitativas de investigación. Estudiaremos la utilidad de esta técnica en el proceso de construcción de los datos. Finalmente, propondremos un balance de la cuestión.
\end{abstract}

Palabras clave: análisis del discurso, metodología, investigación cualitativa, investigación cuantitativa

\section{Introducción}

En este artículo nos proponemos desarrollar una reflexión acerca de las posibilidades metodológicas de una técnica de análisis cuyo estatus y cuya orientación están aún indeterminados: el Análisis del Discurso (AD).

En cuanto al estatus, el $A D$ ha sido definido como un método (Phillips y Jørgensen, Jäger), como una metodología (Powers, Goom) y como una técnica de análisis (Sayago, Santander Molina). Si bien es posible que estas diferencias se deban en parte a cuestiones terminológicas que pueden ser consideradas menores, también hay en juego presupuestos epistemológicos. Tomar el $\mathrm{AD}$ como un método o como una metodología implica asociarlo estrechamente a una clase de relación particular entre la instancia de la construcción teórica y la instancia de la operación empírica, a un vínculo no exento de presupuestos y prescripciones epistemológicas, metodológicas y éticas. También implica asociarlo a una perspectiva específica sobre la construcción y el procesamiento de los datos, definida generalmente como cualitativa (Wood and Kroger, Antaki, Potter, Cheek, Hammersley). 
Tomar el AD como una técnica de análisis, en cambio, acota su estatus metodológico, a la vez que habilita su asociación con diferentes metodologías y perspectivas. Asumimos que esta ductilidad es uno de los atributos que permite considerar al AD una herramienta metodológica superior a la hermenéutica y el Análisis de Contenido (AC).

Para adentrarnos en el tratamiento de este asunto, comenzaremos situando el AD en relación con las otras dos técnicas mencionadas.

\section{La búsqueda del sentido: hermenéutica, análisis de contenido y análisis de discurso}

Las primeras reflexiones lingüísticas sistemáticas se remontan, al menos, hasta el siglo V a.C., cuando los sofistas teorizaron acerca de las técnicas orientadas a la persuasión, inaugurando una vía continuada luego a través de los trabajos de Aristóteles, principalmente en su Retórica, y, en Roma, a través de la Retórica a Herenio y de las obras de Cicerón y Quintiliano. Sin embargo, estas reflexiones estaban acotadas al dominio de la argumentación y tendían más a dar indicaciones para la elaboración de piezas oratorias eficaces que a fijar criterios para el análisis de piezas ya hechas.

Durante la Edad Media, en cambio, se analizó un texto particular, la Biblia, con el fin de reconocer en él sentidos no manifiestos. Este abordaje fue conocido como la hermenéutica religiosa, cuya formulación más completa estuvo a cargo de Santo Tomás, quien, a partir del sentido literal de las Sagradas Escrituras, reconoció tres tipos de sentido espiritual: el alegórico, el moral y el anagógico o místico.

En el siglo XIX, la hermenéutica amplió su área de aplicación desde la exégesis religiosa a la exégesis de los textos escritos en general. Fundando la hermenéutica romántica, Friedrich Schleiermacher propuso un círculo hermenéutico que iba de la reconstrucción del contexto del autor hacia la reconstrucción intuitiva y adivinatoria de su proyecto creador. Este modo de abordaje fue muy difundido sobre todo en el ámbito de los estudios literarios, donde se pensó que una correcta interpretación de los textos debe coincidir con la intención originaria de sus productores, suponiendo la existencia de una lectura única.

Cuando a fines del siglo XIX y principios del XX, empezaron a desarrollarse las ciencias sociales y se hizo necesario estudiar las producciones lingüísticas de los sujetos comunes y corrientes, la hermenéutica romántica se mostró como un instrumento analítico insuficiente, sobre todo a la luz de los estándares científicos dominantes, derivados del modelo de las ciencias naturales. Se necesitaba una herramienta capaz de tratar los textos como objetos, es decir, como productos simbólicos que pudieran ser sometidos a operaciones experimentales y estadísticas.

Esa herramienta fue el AC. Configurado en las primeras décadas del siglo XX en Estados Unidos, primero, fue aplicado al estudio de diarios, en los que se registraba la frecuencia de ciertos temas, la extensión de las noticias, su ubicación en las páginas, etc. Luego, a raíz de la Segunda Guerra Mundial, el análisis fue aplicado también al estudio de la propaganda política. Se prestaba atención a la aparición de ciertas palabras, expresiones o tópicos con el fin de reconocer, dentro del país, publicaciones que propagaran ideologías contrarias a los ideales norteamericanos.

Berelson, el investigador que mejor sistematizó las posibilidades de este nuevo instrumento, lo definió como "a research technique for the objective, systematic, and quantitative description of manifest content of communications" (1952:18).

En los setenta surgieron variantes más blandas, que relativizaron el peso del tratamiento estadístico de los datos para dar cuenta también de los sentidos implícitos en la superficie textual. Así, por ejemplo, Bardin redefinió el propósito del AC como la "inferencia de conocimientos relativos a las condiciones de producción (o eventualmente de recepción), con ayuda de indicadores (cuantitativos o no)" (1986:29). La categoría de inferencia era propuesta como una instancia intermedia, explícita y controlada, en el pasaje de la descripción a la interpretación. 
De este modo, el AC intentaba extender su alcance a las relaciones semánticas y pragmáticas que vinculan el texto con el contexto, es decir, a algo más que el contenido del texto. Pero la interpretación estaba constreñida por el tipo de datos que utilizaba. Una cosa es explicar lo que aparece en un texto o predecir lo que puede aparecer y otra muy distinta es analizar el texto como el producto dinámico de un juego de relaciones sociales en el que los hablantes despliegan estrategias discursivas, explotan el significado implícito de las proposiciones de sus enunciados y en el que los oyentes deben apelar a ciertos conocimientos enciclopédicos para comprender el sentido y la intención de dichos enunciados.

En 1980, consciente de esta tensión epistemológica, Krippendorff reconoció que "quizá la expresión 'análisis de contenido' ya no resulte apropiada para este contexto más amplio, en cuyo interior se entienden (y deben entenderse) los mensajes y los datos simbólicos" (1990:11). Para entonces, había surgido en el campo de las ciencias sociales una herramienta metodológica que amalgamaba desarrollos de la lingüística, de la filosofía, de la sociología, de la antropología, de la historia, de la psicología cognitiva y de la retórica, entre otras disciplinas: el Análisis de Discurso.

Podemos señalar las décadas de 1960 y 1970 como el umbral histórico a partir del cual estas heterogéneas líneas de pensamiento estructuraron un campo interdisciplinario que, además de producir diferentes representaciones de los vínculos entre el lenguaje, el sujeto y la sociedad, elaboró también aplicaciones metodológicas.

Comparado con la hermenéutica y el AC, el AD se presenta como una herramienta más sofisticada, dotada de un aparato conceptual que permite relacionar la complejidad semiótica del discurso con las condiciones objetivas y subjetivas de producción, circulación y consumo de los mensajes. Este aparato puede incluir nociones relativas a los modelos mentales, la identidad, los roles, la polifonía, las estrategias retóricas, las variedades dialectales y estilísticas, los formatos textuales, los géneros discursivos, las ideologías, las relaciones de dominación, etc. A continuación, caracterizaremos metodológicamente el AD.

\section{Aspectos básicos del $A D$ como técnica de análisis}

El AD es, a la vez, un campo de estudio y una técnica de análisis. En tanto campo de estudio, se destaca por su multidisciplinariedad y por la heterogeneidad de corrientes y tradiciones que confluyen en él. No solo está constituido por la convergencia de diferentes ciencias (lingüística, sociología, antropología, psicología social, psicología cognitiva, ciencias políticas, ciencias de la comunicación, pedagogía, etc.), sino que en el interior de cada una de esas ciencias, pueden converger corrientes muy distintas entre sí. Pensemos, por ejemplo, en la coexistencia, dentro de la lingüística aplicada al $A D$, de la teoría de la enunciación (de tradición francesa) y de la lingüística funcional (de tradición anglosajona), advirtiendo, además, que dentro de cada una de ellas hay variadas propuestas teóricas.

Esta multiformidad heteróclita de la instancia de la producción teórica conspira contra la estandarización del AD como técnica de análisis. Tal como enfatiza Santander, "no existe la técnica para hacer el análisis" (2011:215). Estamos ante una caja de herramientas lo suficientemente vasta como para experimentar tanto desazón como entusiasmo.

En cada investigación, el problema de la heterogeneidad teórica se puede resolver mediante la atenta observación de un criterio de pertinencia y de rigurosidad que guíe la selección de las categorías apropiadas y el diseño de los procedimientos de análisis. Sin embargo, este ejercicio de reflexión y vigilancia teórica no siempre se lleva a cabo (con éxito) y abundan los análisis discursivos que, en realidad, son resúmenes, comentarios redundantes o injustificados, análisis de citas fragmentarias y descontextualizadas o un mero registro de recursos lingüísticos extraídos del corpus.

Pese a este riesgo, como intentaremos demostrar, el $A D$ es una técnica de análisis potente y precisa, que resalta por su ductilidad. Por un lado, puede ser utilizada por metodologías hipotético-deductivas, inductivas y abductivas (según la clasificación de Samaja). En las primeras, la instancia de la construcción teórica precede a la instancia del trabajo empírico; en las segundas, la instancia del trabajo empírico precede a la instancia de la construcción teórica; en la última, ambas instancias se desarrollan paralelamente, en un constante movimiento de ida y vuelta. 
Por otro lado, puede formar parte de estrategias cualitativas y de estrategias cuantitativas. Aquí, exploraremos esta segunda posibilidad, la cual resulta particularmente interesante dado que el AD suele quedar preso de una exagerada división de aguas entre lo cualitativo y lo cuantitativo. Esta dicotomía, frecuentemente planteada como una separación de metodologías o de métodos o incluso de paradigmas, puede simplificar la productividad de esta herramienta. Con el objetivo de hacer un aporte orientado a reforzar la evidencia de la intrínseca complementariedad de las dos caras de la misma moneda, haremos un ejercicio de aplicación del AD al estudio de un mismo objeto, utilizando, primero, una estrategia cualitativa y, luego, una cuantitativa.

Antes de continuar, vale aclarar que es común recurrir al AD como técnica de análisis por dos razones: a) porque lo pide el objeto de estudio, es decir, porque es el modo más adecuado para su análisis o b) porque decidimos realizar un trabajo de análisis del discurso y, entonces, partimos de la elección de la técnica de análisis y, luego, escogemos un tema que se ajuste a las posibilidades que esta técnica nos abre. La primera razón es estrictamente metodológica, en tanto se desprende de los requerimientos propios de un proceso de investigación en curso. La segunda razón está relacionada más globalmente con la práctica de investigación en general, como un modo de desarrollar y ejercitar una experticia teórico-metodológica particular.

Si bien ambas son igualmente legítimas, escogeremos la primera razón, que es la más conveniente de acuerdo con los objetivos de este artículo. Supongamos que estamos en la instancia de construcción del objeto de estudio y, valiéndonos de intuiciones, de nociones pre-teóricas, de categorías teóricas y de reflexiones acerca de nuestra praxis social, elaboramos la representación de una determinada clase de hechos. Si el resultado es la construcción idealizada de un evento comunicativo en el que el espacio, la vestimenta y las actitudes corporales son fundamentales, seguramente recurriremos a la observación etnográfica como técnica de recolección de datos y al análisis de los registros elaborados como técnica de análisis.

$\mathrm{Si}$, en cambio, lo que vamos a estudiar es la representación discursiva de esos eventos comunicativos en cierta clase de revistas, la selección de publicaciones (ya sea por criterios de estudios de caso o por muestreo) será la técnica de recolección de datos utilizada y el $A D$, la técnica de análisis más apropiada. En algunas investigaciones, dados los objetivos propuestos, es recomendable e incluso necesario el uso complementario de dos o más técnicas de recolección de datos y también de dos o más técnicas de análisis.

Supongamos que queremos analizar el modo en que la prensa escrita chilena representa un acontecimiento particular: las acciones de resistencia de una comunidad específica ante un emprendimiento minero que amenaza su forma de vida. Más específicamente, queremos estudiar el discurso de los principales medios de prensa a través de sus relatos noticiosos (crónicas) de este acontecimiento puntual.

Decidimos hacer un estudio comparativo entre medios considerados conservadores y medios considerados críticos. Con el fin de acotar la cantidad de medios a estudiar, además del criterio de orientación ideológica, de manera complementaria podemos tomar dos más: tirada (cantidad de impresos) y relevancia social o política. Supongamos que, de la aplicación de estos tres criterios, resulta la selección de dos diarios online y dos portales de noticias. Luego, buscamos todas las crónicas publicadas sobre este tema y las ordenamos cronológicamente. De este modo, construimos la serie noticiosa.

Una primera lectura del corpus nos permitirá extraer algunos datos sencillos: cantidad de crónicas producidas por cada medio, frecuencia de publicación, grado de relevancia otorgado, extensión, uso de polifonía (predominio de citas de autoridades gubernamentales, de miembros de la comunidad, de representantes de la empresa, etc.). Esta información es muy útil y, en numerosas ocasiones, es todo lo que se busca en una investigación. Pero, si queremos estudiar las representaciones discursivas puestas en circulación por la prensa, tenemos que profundizar en la búsqueda de elementos que nos permitan reconstruir la matriz de sentido (Pêcheux) que las articula. Para ello, el AD es una herramienta muy útil. 
Vale aclarar que estamos proponiendo un estudio de caso. La selección del caso debe ser justificada por criterios teóricos tales como: tipicidad, discrepancia, reputación teórica, etc. (Goetz y Le Compte). Sin embargo, en vez de un estudio de caso, podríamos haber elegido un muestreo según criterios estadísticos. Esta posibilidad no es la más conveniente para estudios que pretenden estudiar el tratamiento mediático de un acontecimiento específico. El muestreo estadístico es más apropiado para la selección de textos pertenecientes a una sección fija del diario, por ejemplo.

\section{El AD como parte de una estrategia cualitativa}

Exploremos, primero, las posibilidades que ofrece el AD como una herramienta de análisis cualitativo. Podemos analizar las representaciones discursivas puestas en circulación por cada medio, centrando la atención en categorías tales como la justificación del conflicto, la descripción de los hechos, la caracterización de los actores sociales involucrados, el tono del relato, la importancia otorgada a los aspectos ecológicos, económicos y culturales del emprendimiento minero, la expresión de expectativas acerca de las consecuencias del conflicto a corto, a mediano y a largo plazo, la referencia al rol del gobierno y al del Estado. Tratamos de reconocer estas categorías en las crónicas publicadas por cada medio, atendiendo a los desplazamientos que pudieran ocurrir durante el desarrollo de la serie.

Tomaremos la crónica como el texto mediante el cual se concreta el discurso noticioso de cada medio. Seguimos aquí una concepción de discurso que lo define como un texto (oral y/o escrito) más todos los aspectos relevantes en la instancia de su producción, de su circulación y de su recepción. Este concepto puede ser expresado mediante la fórmula Discurso = Texto + Contexto (Lavandera). Así, para estudiar el discurso de un diario determinado, hay que analizar los textos mediante los cuales este discurso se realiza. La crónica es la clase textual que típicamente expresa el relato noticioso de cada medio.

Cada crónica será, entonces, una unidad de análisis (UA) en la que podremos reconocer diferentes variaciones entre los discursos de cada medio e incluso variaciones dentro del discurso de un mismo medio. Así, podríamos advertir que algunos medios no profundizan en las causas del conflicto o en las consecuencias ecológicas de la producción minera y sí, en cambio, realzan la importancia de las acciones físicas de los manifestantes de la comunidad o de las explicaciones del gobierno o de los representantes de la empresa. Otros medios, en cambio, analizan con mayor cuidado las causas del conflicto, caracterizan positivamente a los personajes comunitarios y cuestionan el rol de gobierno en el conflicto y el rol del Estado en el contexto más amplio de la política minera nacional.

El análisis comenzaría con un proceso de codificación consistente en el etiquetamiento y la desagregación de pasajes textuales de acuerdo con la categoría buscada. El etiquetamiento o rotulación es la identificación de un pasaje como realización de una categoría determinada. La desagregación es la extracción de estos pasajes.

Vale advertir que un mismo fragmento puede ser incluido en más de una categoría y que la búsqueda puede ser tanto vertical como transversal. La búsqueda vertical trata de reconocer todas las categorías propuestas que están presentes en cada UA. La búsqueda transversal privilegia el reconocimiento de una misma categoría en las diferentes UAs. Así, por ejemplo, si hubiéramos seleccionado para el análisis las categorías a) justificación, b) descripción del conflicto y c) caracterización de los personajes, en la búsqueda vertical intentaríamos reconocer pasajes que realicen estas categorías en cada texto. Tomaríamos el Texto $\mathrm{N}^{\circledR} 1$ y seleccionaríamos uno o más pasajes como realización de la categoría justificación, uno o más pasajes como realización de la categoría descripción del conflicto y uno o más pasajes como realización de la categoría caracterización de los personajes.

En una búsqueda transversal, intentaríamos reconocer, en todos los textos, primero, la realización de la categoría justificación, luego la realización de la categoría descripción del conflicto y, por último, la realización de la categoría caracterización de los personajes. 
Una vez que señalamos mediante etiquetas los fragmentos, estos son extraídos de las UA y son reagrupados en un nuevo texto, el que nos permitirá reconocer semejanzas y diferencias. Podemos utilizar subetiquetas para marcar estos matices y para subespecificar cada categoría.

Este proceso de etiquetamiento-desagregación-reagregación es impulsado por una tarea de interpretación que debe ser altamente reflexiva, ya que es necesario evaluar de manera constante la validez de las semejanzas y diferencias reconocidas. Debemos tener en cuenta, de acuerdo con los objetivos de la investigación, hasta qué punto conviene señalar las diferencias y hasta qué punto conviene ignorarlas. También debemos esmerarnos en encontrar correspondencias entre los sesgos valorativos, con el fin de reconstruir las matrices de sentido que articulen las distintas representaciones discursivas.

Para realizar un estudio como este, no es necesario recurrir a procedimientos estadísticos. Basta con construir los datos, procesarlos e interpretarlos.

\section{El AD como parte de una estrategia cuantitativa}

La elección de una estrategia de análisis cualitativa o cuantitativa depende de los objetivos y de la tradición en la que el investigador se inscribe o desea inscribirse. Podríamos haber decidido realizar un estudio cuantitativo del mismo objeto (las representaciones discursivas de la prensa escrita relativas al proceso de resistencia de una comunidad frente a un emprendimiento de minería). Habríamos construido los datos de un modo tal que nos permita someterlos a un procesamiento estadístico. Los aspectos que constituyan las variables a considerar deberían ser discretos, es decir, tener límites definidos.

Hemos establecido anteriormente que el discurso de los medios se materializa en diferentes clases de textos, siendo una de ellas la crónica, la que constituye nuestra principal unidad de análisis (UA). Ahora, debemos reconocer y seleccionar las variables (Vs) más relevantes de acuerdo con nuestros objetivos. Supongamos que seleccionamos las tres categorías planteadas anteriormente (en el contexto de desarrollo de una estrategia cualitativa): justificación del conflicto (V1), descripción el conflicto (V2) y caracterización de los personajes (V3). Para cada una de ellas, deberíamos definir un tipo de valor (R) y un indicador (I), que es el procedimiento mediante el cual podremos medir el comportamiento de cada variable. Veamos los valores posibles para cada variable:

Figura 1: Matriz de datos de la UA crónica

\begin{tabular}{|c|c|c|c|}
\hline UA & V1: justificación del conflicto & V2: descripción del conflicto & $\begin{array}{c}\text { V3: caracterización de los } \\
\text { personajes }\end{array}$ \\
\hline crónica & R: superficial/media/profunda & R: general/media/detallada & R: negativa/neutra/positiva \\
\hline
\end{tabular}

Fuente: Elaboración propia

La escala de medición de estas variables es ordinal. El valor de V1 podría depender de la cantidad y la solidez de las causas tratadas. Así, una justificación que desarrolle con rigurosidad los argumentos que explican el conflicto sería considerada profunda, una que desarrolle pocos argumentos será considerada media y una que apenas mencione las causas o motivos del conflicto y/o que los desarrolle pero de un modo endeble sería considerada superficial.

Podemos dar un paso más y medir cuantitativamente la solidez de las justificaciones. Cada encadenamiento argumentativo (EA) puede ser ponderado de acuerdo con su solidez argumentativa. Cuantificaremos esta variable, dando un valor entre 0 y 6 , dependiendo de la validez del vínculo entre el argumento y la conclusión. El valor superficial corresponde al rango de 0 a 2, el valor media al de 2,1 a 4 y el valor profunda al de 4,1 a 6. 
Para la asignación de estos valores se pueden tener en cuenta los siguientes rasgos (rs):

-r1: pertinencia semántico-proposicional: relación entre la información dada como argumento y la información dada como consecuencia de ese argumento;

-r2: claridad estilistica: formulación ordenada y relativamente sencilla de una idea;

-r3: efecto retórico: orientación expresiva al logro de un impacto emotivo y conceptual.

A cada uno de estos rasgos, le asignaremos un valor entre 0 y 2 . Así, si la relación semántico-proposicional entre el argumento y la conclusión es poco pertinente, recibe un valor bajo y si, en cambio, es muy pertinente, un valor alto. Por ejemplo, dado el siguiente EA:

(a) Los pobladores protestan porque se suman a los movimientos de resistencia organizados en muchas zonas del país (1).

Podemos reconocer una relación poco pertinente entre el argumento (se suman a los movimientos de resistencia organizados en muchas zonas del país) y la conclusión (los pobladores protestan). El grado de pertinencia es baja porque ese argumento explica muy débilmente cuáles son los motivos por los que estos personajes (los pobladores) deciden actuar. La acción colectiva es representada como parte de una tendencia generada como una moda. Comparemos con el próximo EA:

(b) Los pobladores protestan porque asumen que el emprendimiento minero afectará la disponibilidad del recurso hídrico necesario para sus actividades agrícolas y para su vida misma.

En este caso, se atribuye a los personajes una motivación plausible para la realización de las acciones de protesta. Por lo tanto, en la escala de 0 a 2, podemos darle un valor alto a esta relación semántico-proposicional.

Vale aclarar que, si nuestro único objeto de estudio es el de las representaciones discursivas de la prensa, lo que estudiamos es precisamente eso: representaciones discursivas. No estudiamos toda la realidad, sino la representación de la realidad construida por el sistema de medios de comunicación social (representación que forma parte de la realidad del investigador, claro). La ponderación de rasgos tales como la pertinencia semántico-proposicional se establece de acuerdo a los criterios de verosimilitud que la prensa construye y negocia a través de variados recursos de polifonía. Por esta razón, podemos afirmar que el EA (b) presenta un vínculo plausible entre el argumento y la conclusión, pero no podemos afirmar que este sea verdadero o falso. Para decidir la adecuación empírica de este EA (es decir, su verdad o su falsedad), deberíamos complementar el estudio de las representaciones de la prensa con un estudio sociológico y antropológico de la comunidad afectada. Entonces, sí estaríamos en condiciones de afirmar que el EA producido por un medio particular describe adecuadamente (o no) lo que sucede en esa organización social.

Resumiendo lo hecho en este punto, para asignar los valores superficial/media/profunda al comportamiento de la V1 justificación del conflicto, hemos definido tres rasgos para los EAs que la conforman: pertinencia semánticoproposicional $(\mathrm{r} 1)$, claridad estilística $(\mathrm{r} 2)$ y efecto retórico $(\mathrm{r} 3)$. Cada EA puede recibir un valor numérico máximo de 6, pudiendo aportar cada rasgo un máximo de 2.

Así, mientras más EAs conformen una justificación y mientras mejor sean satisfechos los rasgos seleccionados, mayor será el valor numérico de V1, lo que permitirá traducir la escala cuantitativa a la escala cualitativa (superficial/media/profunda). La escala ordinal es construida sobre una escala de intervalo.

Mediante estas investigaciones podremos determinar si las representaciones discursivas de cada medio son precisas o imprecisas. 
Las unidades que hemos definido están organizadas jerárquicamente, por lo que es posible representarlas en palabras de Samaja en un "sistema de matrices de datos" (1994:178).

Figura 2: Sistema de matrices de datos

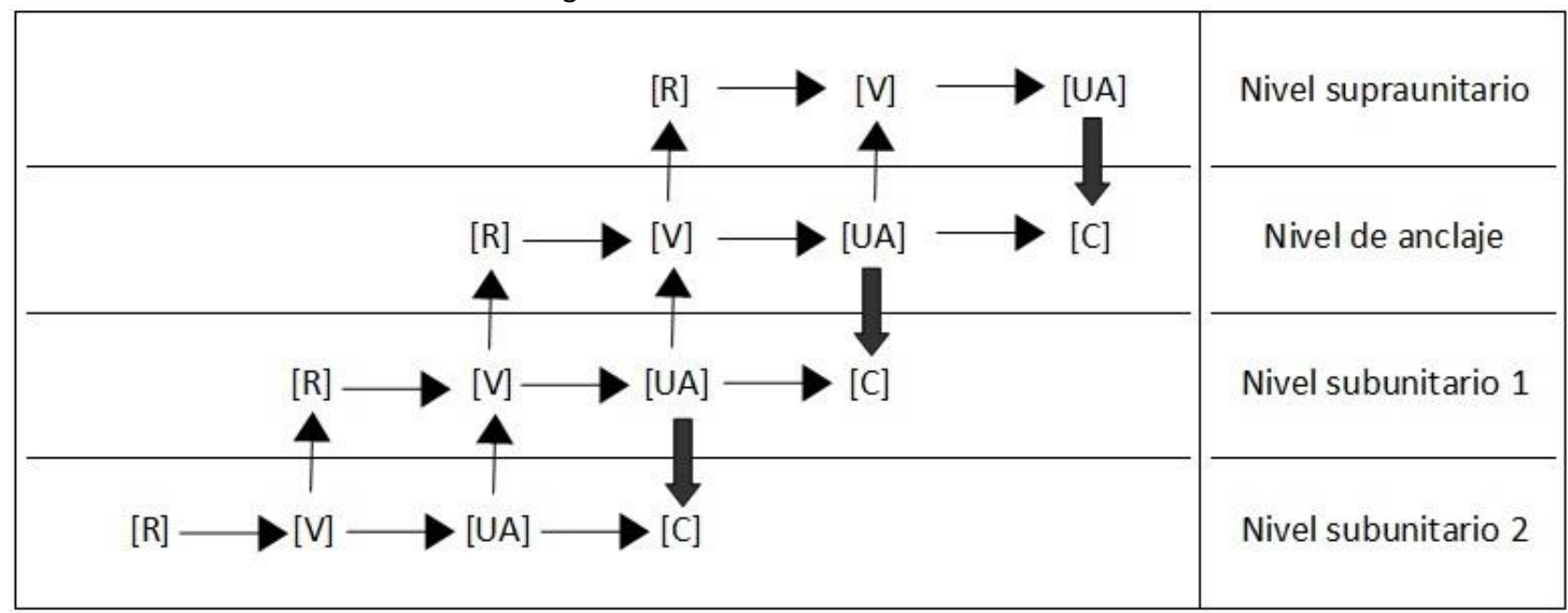

Fuente: Reelaboración del esquema propuesto por Samaja (1994:178). Se ha agregado un segundo nivel subunitario. Este cambio no contradice la postura epistemológica y metodológica de Samaja.

Cada nivel se especifica en un nivel inferior. La unidad de análisis (UA) queda reespecificada como contexto (C), la variable (V) como UA y el valor (R) como variable (V). Si leemos el esquema de manera ascendente, el valor (R) y la variable $(V)$ del nivel inferior constituyen el valor (R) del nivel superior, la unidad de análisis (UA) del nivel inferior constituye la variable (V) del nivel superior y la unidad de análisis (UA) del nivel inferior constituye la variable (V) del nivel superior.

Hemos definido como el nivel de anclaje el que corresponde a la crónica como UA. También hemos asumido la existencia de un nivel superior o superunitario, el que corresponde al discurso del medio como UA. En este nivel, el relato noticioso es solo una de las variables (otras variables pueden ser: entretenimiento, reportajes, publicidad, avisos clasificados, etc.). Distinguimos tres variables (Vs) de la UA del nivel de anclaje: la justificación del conflicto, la descripción del conflicto y la caracterización de los personajes. A los efectos expositivos, avanzamos con la especificación de la primera de ellas.

Descompusimos el nivel de anclaje en dos niveles subunitarios. En el primero, la crónica es el C y la UA, la justificación del conflicto. La $V$ seleccionada es la solidez del EA, cuyo R es un puntaje entre 0 y 6 . Luego, descompusimos este nivel y llegamos a un segundo nivel subunitario. Aquí, la justificación del conflicto es el C, la UA es el EA, la V la relación semántico-proposicional y el $R$ un puntaje entre 0 y 2 .

Pasamos en limpio las matrices de cada nivel: 
Figura 3: Matrices por niveles

\begin{tabular}{|c|c|c|c|}
\hline $\begin{array}{c}\text { Nivel } \\
\text { supraunitario }\end{array}$ & $\begin{array}{c}\text { Nivel } \\
\text { de anclaje }\end{array}$ & $\begin{array}{c}\text { Nivel } \\
\text { subunitario } 1\end{array}$ & $\begin{array}{c}\text { Nivel } \\
\text { subunitario } 2\end{array}$ \\
\hline UA: discurso del medio & C: discurso del medio & C: crónica & C: justificación del conflicto \\
\hline V: crónica (relato de & UA: crónica & UA: justificación del & UA: EA \\
\hline hechos noticiosos) & V: justificación del conflicto & conflicto & V: relación semántico- \\
\hline R: imprecisa/precisa & R: superficial/media/ & V: solidez del EA & proposicional \\
\hline & profunda & R: $0-6$ & R: $0-2$ \\
\hline
\end{tabular}

Fuente: Elaboración propia

A continuación, reorganizamos estos elementos según el esquema del sistema de matrices de datos:

Figura 4: Sistema de matrices de datos del análisis del relato noticioso de la prensa

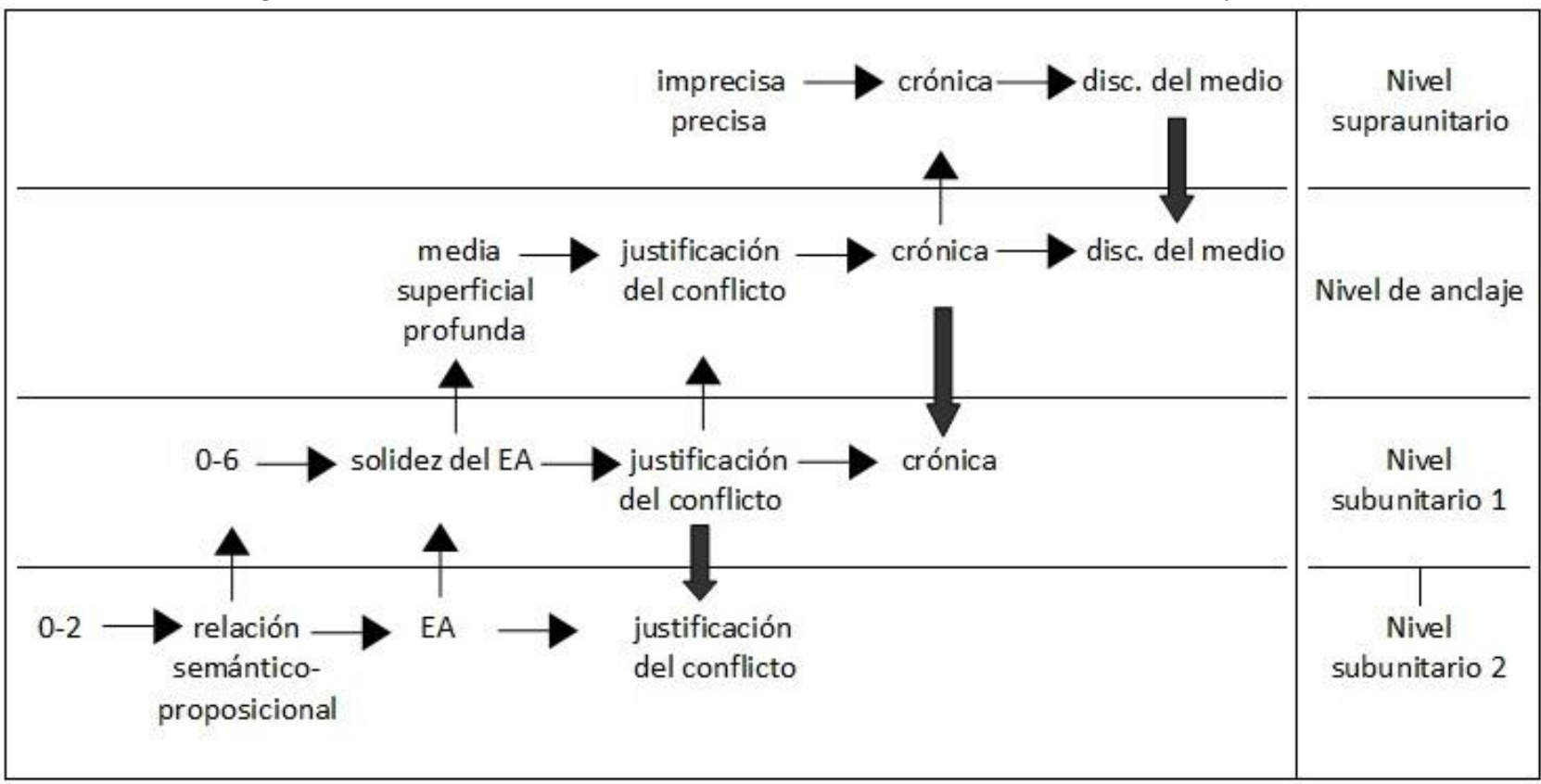

Fuente: Elaboración propia

La cuantificación mediante escala de intervalo del nivel subunitario 2 sustenta la cuantificación mediante escala de intervalo en el nivel subunitario 1. Hemos establecido una escala ordinal en el nivel de anclaje y en el nivel supraunitario, aunque podríamos haber utilizado también una escala de intervalo.

Podemos realizar un procedimiento de especificación similar con las otras variables del nivel de anclaje: descripción del conflicto (V2) y caracterización de los personajes (V3).

En el caso del AD, la cuantificación no supone de ningún modo un proceso automático, ya que requiere una constante tarea de interpretación. Hay una dimensión hermenéutica que atraviesa el proceso de medición en todos los niveles. Esto es algo que diferencia al $A D$ del $A C$, ya que esta última es una técnica cuya aplicación, al menos en algunas instancias, es efectivamente automática. 


\section{Conclusión}

Lo expuesto hasta aquí nos permite afirmar que el AD es una técnica de análisis que puede ser utilizada tanto en una investigación cualitativa como en una cuantitativa. Contra lo que parece instalado en el sentido común de una parte de los investigadores de las ciencias sociales, la precisión conceptual del AD no está reñida con la cuantificación. Hemos visto que es posible traducir, con minuciosidad, al lenguaje matrices de datos (Samaja 1994) distintos rasgos de una representación discursiva. La elección de esta técnica como parte de estrategias cualitativas o de estrategias cuantitativas responde, principalmente, a los objetivos y a la tradición disciplinar de la que el investigador forma parte.

También hemos señalado que el $A D$ no es una técnica que empareje de modo automático un aspecto del objeto estudiado con un valor de una variable, porque, entre ambos componentes, tiene lugar una ineludible tarea de interpretación. Esta tarea está regulada por la coherencia teórica de las nociones que, provenientes de diferentes disciplinas, contribuyen a generar una sofisticada conceptualización de los discursos estudiados. En el ejemplo de análisis que desarrollamos, hemos presupuesto como base para el proceso de interpretación conceptos teóricos provenientes de teorías de la comunicación social y de teorías de la argumentación.

En resumen, la complejidad teórica del AD se manifiesta en la cantidad de nociones que pueden constituir categorías y subcategorías, unidades de análisis y variables. Con mayor potencia heurística que la hermenéutica y el AC, es un instrumento útil para elaborar mapas con la pretensión de aprehender ese territorio que siempre nos desafía.

\section{Nota}

(1) Este es un caso de organización retrogresiva de la secuencia argumento-conclusión, porque la conclusión aparece en primer lugar y el argumento en segundo lugar, luego del operador argumentativo porque. Cuando el argumento antecede a la conclusión, la organización es progresiva.

\section{Bibliografía}

Bardin, L. 1986. El análisis de contenido. Madrid: Akal.

Berelson, B. 1952. Content analysis in communications research. Glencoe: The Free Press.

Krippendorff, K. 1990. Metodología de análisis de contenido. Barcelona: Paidós.

Samaja, J. 1994. Epistemología y metodología. Buenos Aires: Eudeba.

Santander, P. 2011. Por qué y cómo hacer análisis de discurso. Cinta moebio 41:207-224.

Recibido el 23 Jul 2013

Aceptado el 15 Dic 2013 